\title{
Estudio de la entrada de agua en pinturas de base agua: comparación entre pintura aplicada y libre
}

\author{
C. Pérez ${ }^{(*)}$, A. Collazo $^{(*)}$, M. Izquierdo ${ }^{(* *)}$, P. Merino $^{(*)}$ y X.R. Nóvoa ${ }^{(* *)}$
}

\begin{abstract}
Resumen Uno de los mecanismos de actuación de las pinturas como protectoras de metales frente a la corrosión es como barreras más o menos eficaces entre el medio y el metal. En el presente trabajo se estudia la entrada de agua en una pintura acrílica de base agua como película libre y como película aplicada sobre un sustrato inerte, empleando una disolución de cloruro sódico al $5 \%$. Las medidas de espectroscopía de impedancia ( $E I)$ llevadas a cabo han permitido observar el importante incremento de la capacidad de la pintura con el tiempo de exposición que, mediante la expresión de Brasher y Kingsbury, se puede correlacionar con la fracción de volumen de agua que entra en la película. Se ha podido establecer que la difusión no es ideal fickiana y se han determinado los coeficientes de difusión aparente. Los resultados apuntan a diferencias poco significativas entre ambas situaciones.
\end{abstract}

Palabras clave: Entrada de agua. Pintura base agua. Coeficiente de difusión. Espectroscopía de impedancia. Constante dieléctrica.

\section{Water uptake in a water-borne paint: a supported and free film comparison}

\begin{abstract}
Paints behave as barriers to protect the metallic substrate against corrosion. In the present work, the water uptake in an acrylic water-borne paint was studied under two different conditions: supported and free film, inmersed in a sodium chloride $5 \%$ solution. Using the impedance spectroscopy (IS) technique, a sharp increase in the film capacitance was observed, which is related to the volume fraction of water taken into the film by using the Brasher-Kinsbury equation. The results suggested that there was no big differences between both situations. The calculated apparent diffusion coefficient suggests that the difference between both tested situations is of minor importance.
\end{abstract}

Keywords: Water uptake. Water-borne paint. Diffusion coefficient. Impedance spectroscopy. Dielectric constant.

\section{INTRODUCCIÓN}

La entrada de agua en una pintura introduce cambios importantes en sus propiedades dieléctricas, por lo que, en los últimos años, numerosos investigadores han estudiado este fenómeno empleando la técnica de espectroscopía de impedancia $(E I)$, bien sea sobre películas de pintura libres $(1 \mathrm{y}$ 2) o aplicadas sobre sustratos metálicos (3-6). El

(*) Dpto. de Ingeniería de Materiales, Mecánica Aplicada y Construcción. E.T.S. Ing. Ind. y de Minas, Univ. de Vigo. Lagoas Marcosende 9. 36310-Vigo (España).

(**) Dpto. de Ingeniería Química. E.T.S. Ing. Ind. y de Minas, Univ. de Vigo. Lagoas Marcosende 9. 36310-Vigo (España). objetivo de este trabajo es efectuar un estudio comparativo entre la película libre y aplicada sobre un soporte inerte y, por tanto, en ausencia de reacciones electroquímicas.

En general, la evolución típica de los valores de capacidad obtenidos mediante EI a lo largo del tiempo de exposición muestran un período inicial en el que se produce un incremento importante de la misma hasta llegar a un determinado valor $\mathrm{C}$, en el que se estabiliza, como consecuencia de la saturación de la película (7 y 8 ).

Teniendo en cuenta que la constante dieléctrica del agua (a $20^{\circ} \mathrm{C}$ su valor es 80 ) es mucho mayor que la correspondiente al polímero seco (con valores que oscilan entre 2 y 5 ), es lógico pensar que el 
incremento de la capacidad de la película con el tiempo de exposición, sea debido a la entrada de agua en la misma. Se han desarrollado numerosas ecuaciones para cuantificar esa relación. Lindqvist (9) ha hecho un estudio comparativo de las seis ecuaciones más utilizadas, las de Boettcher, Bruggeman, Looyenga, Wagner, Rayleign, y BrasherKingsbury, llegando a la conclusión de que esta última es la que mejor se ajusta al método gravimétrico. La ecuación de Brasher y Kinsbury (10), propuesta ya en los años cincuenta, establece la siguiente relación empírica entre la capacidad de la pintura y la fracción de agua en volumen que entra en la pintura:

$$
X_{\mathrm{v}}(\%)=\frac{\log \left(C_{\mathrm{t}} / C_{\mathrm{o}}\right)}{\log \varepsilon_{\mathrm{H}_{2} \mathrm{O}}} \cdot 100
$$

donde $X_{v}$ es la fracción, en volumen y en porcentaje, de agua que entra en la película; $C_{t}$ y $C_{o}$ son la capacidad de la pintura a tiempo $t$ y a tiempo $t=0$, respectivamente, y $\varepsilon_{\mathrm{H}_{2} \mathrm{O}}$ es la constante dieléctrica del agua pura. Esta ecuación es la más ampliamente utilizada para el cálculo de la entrada de agua en las pinturas (11 y 12).

A partir de los datos de la cantidad de agua absorbida por la pintura, para cortos períodos de tiempo, y suponiendo un proceso de difusión ideal, es posible, obtener el valor del coeficiente de difusión, $D$, si la entrada de agua varía linealmente con $\sqrt{t}(13)$.

\section{METODOLOGÍA}

La pintura empleada era una resina acrílica de base agua, pigmentada con óxido de hierro micáceo (MIO) y óxido de hierro rojo, con un contenido de pigmentos en volumen (CPV) del $33 \%$.

Las muestras de película libre, con un espesor medio $81 \mu \mathrm{m}$, se obtuvieron al desprender la pintura del soporte sobre el que fueron aplicadas. $\mathrm{Pa}$ ra las muestras de película aplicada, de espesor $160 \mu \mathrm{m}$, se utilizó como soporte inerte polietileno comercial (PE) rígido de $170 \mu \mathrm{m}$ de espesor. En las dos muestras, se puso en contacto una de las caras de la película con una disolución de $\mathrm{NaCl}$ al $5 \%$.

Las medidas de impedancia se llevaron a cabo empleando el analizador de impedancia HP-4194a, que permite la medida de la capacidad en el intervalo de $10^{-14} \mathrm{~F}$ a $0,1 \mathrm{~F}$, con una resolución máxima de $10^{-16} \mathrm{~F}$. La celda de medida estaba formada por dos láminas cuadradas de aluminio de $9 \mathrm{~cm}^{2}$ de superficie, entre las que se colocó la pintura, en una disposición tipo sandwich.

Los valores de impedancia calculada se obtuvieron a partir de la simulación del proceso mediante circuitos equivalentes, consistentes en diversas combinaciones de elementeresistivos y capacitivos, que se detallan en el apartado 3.1. El ajuste se basó en el algoritmo desarrollado por Nelder y Mead (14) que usa el método simplex para encontrar el mínimo de la función de ajuste.

\section{ANÁLISIS Y DISCUSIÓN DE RESULTADOS}

\subsection{Evolución de la capacidad}

Para el caso de la pintura aplicada, el sistema estaba constituido por dos capas: plástico soporte y película de pintura; la impedancia total puede considerarse, por lo tanto, como la suma en serie de

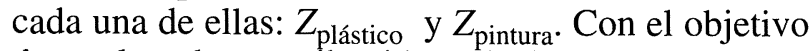
de evaluar la contribución del plástico a la impedancia total, se sometió, únicamente a éste, a las mismas condiciones de ensayo que todo el conjunto. Como cabía esperar, su comportamiento fue típicamente cápacitivo durante todo el ensayo (Fig. 1). El circuito empleado fue un $R C$ en serie (Fig. 1), donde $R_{\mathrm{o}}$ representa la resistencia asociada a las conexiones externas y $C_{\mathrm{p}}$ la capacidad del plástico. El valor medio de la capacidad fue de $10 \mathrm{pF} / \mathrm{cm}^{2}$, el cual no ha sufrido cambios significativos a lo largo del ensayo, lo que nos permite concluir que la aportación del plástico a la impedancia total será un valor constante, que es posible restar de la impedancia total del sistema.

Los espectros de impedancia obtenidos tanto para la pintura aplicada como para la libre (las figuras 2 y 3 , respectivamente) muestran inicialmente un comportamiento puramente capacitivo $\mathrm{y}$, a medida que aumenta el tiempo de exposición, tiene lugar

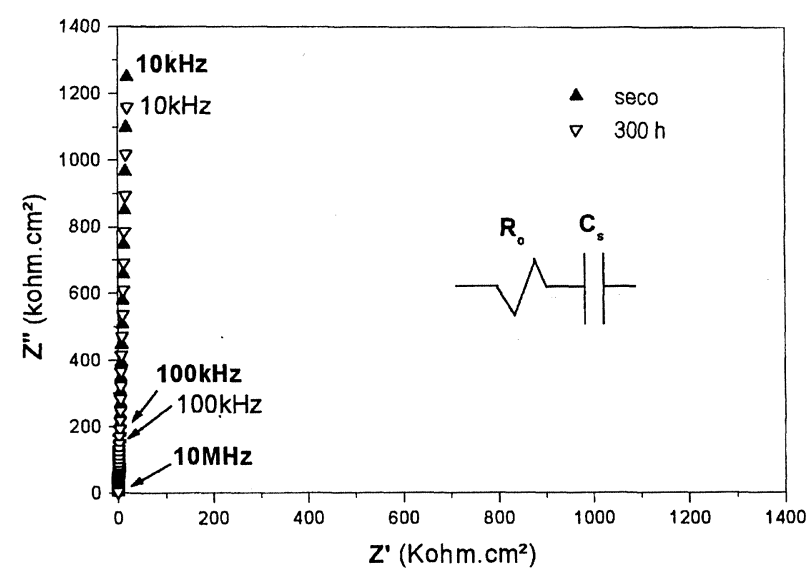

FIG. 1.- Diagramas de Nyquist del plástico soporte y el circuito equivalente empleado en la simulación.

FIG. 1.- Nyquist diagram corresponding to the mounting plastic and equivalent circuit employed for simulation. 


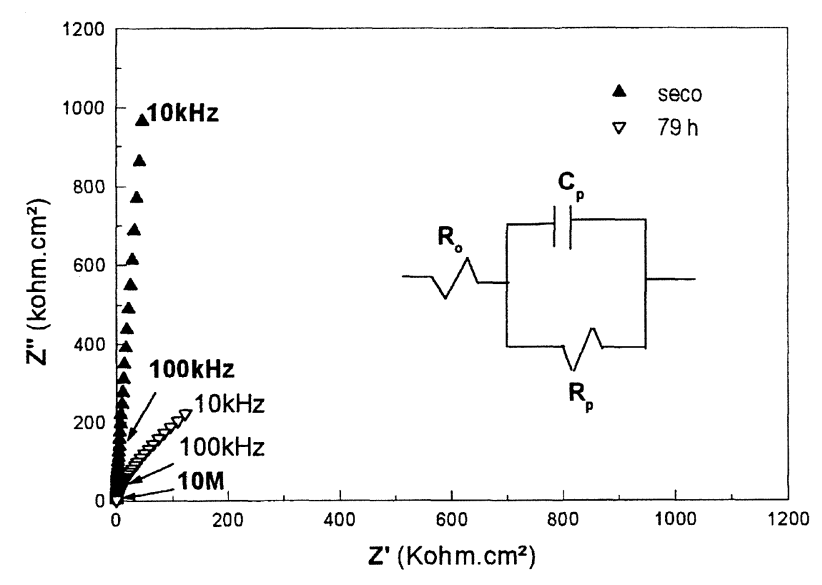

FIG. 2.- Diagramas de Nyquist de la película aplicada y el circuito equivalente correspondiente.

FIG. 2.- Nyquist diagram corresponding to the applied film for dry condition (up triangle) and after $79 \mathrm{~h}$ immersion (down triangle). The equivalent circuit employed for simulation is also depicted.

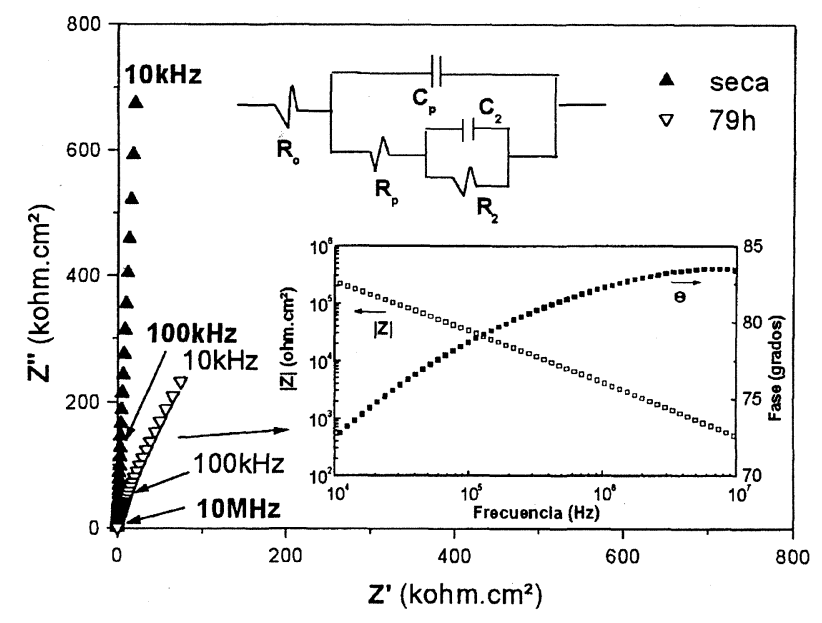

FIG. 3.- Diagramas de Nyquist y Borde de la película libre, junto con el circuito equivalente empleado para la simulación.

FIG. 3.- Nyquist diagram corresponding to the free film for dry condition (up triangle) and after $79 \mathrm{~h}$ immersion (down triangle). The equivalent circuit employed for simulation is also depicted.

un descenso muy acusado de la impedancia. Los diagramas experimentales obtenidos para la pintura aplicada pueden ajustarse empleando un circuito equivalente $R C$ en paralelo, donde $R_{\mathrm{o}}$ tiene el mismo significado que en el caso del plástico soporte y $R_{\mathrm{p}}$ y $C_{\mathrm{p}}$ son los valores de la resistencia y la capacidad asociados a la pintura. En el caso de la película libre, sin embargo, aparece una segunda constante de tiempo a frecuencias más bajas, por lo que para el ajuste de los datos experimentales se ha utilizado un modelo de dos RC en cascada. La primera de las constantes de tiempo se ha asignado al comportamiento dieléctrico de la película, de forma análoga a la película aplicada. La interpretación de la segunda constante de tiempo es más compleja. A nuestro juicio, puede deberse a la existencia de la interfase entre la lámina de aluminio y la pintura, que únicamente se pone de manifiesto cuando la película de pintura se encuentra suficientemente húmeda.

En la evolución de los valores de $R_{\mathrm{p}}$ y $C_{\mathrm{p}}$ (Fig. 4 ), se puede observar un importante incremento en el valor de la capacidad con el tiempo de inmersión, hasta llegar un momento, $t_{s}$ (tiempo de saturación), en que su valor se estabiliza. Esta situación se corresponde con la saturación de la película, momento a partir del cual se establece un equilibrio dinámico entre la cantidad de electrólito que entra y la que sale de la película.

Mediante la relación de Brasher y Kinsbury (ec. [1]) se puede relacionar este aumento de la capacidad, $C_{\mathrm{p}}$, con la cantidad de agua que entra en la pintura a lo largo del tiempo de inmersión (Figs. 5 y 6). Como era de esperar, en ambos sistemas, la entrada de agua en las primeras horas de exposición es mucho más importante, hasta alcanzar el período de estabilización o saturación de la pintura. El porcentaje de agua absorbida es análogo en ambos casos; sin embargo, el tiempo de saturación, es marcadamente distinto: $9,5 \mathrm{~h}$ en la película aplicada y sólo $5 \mathrm{~h}$ en la película libre.

Es posible ajustar el primer período a una ecuación exponencial, $y=A x^{\mathrm{n}}$ (Figs. 5 y 6 ). El valor del exponente $n$ obtenido para la película libre es 0,36 y para la película aplicada, 0,41 . Ninguno de ellos se corresponde con el de una difusión ideal fickiana. Este resultado no es sorprendente si se tiene en cuenta la enorme complejidad que entraña una pintura, donde existen numerosos constituyentes de naturaleza distinta (resinas, pigmentos, aditivos,

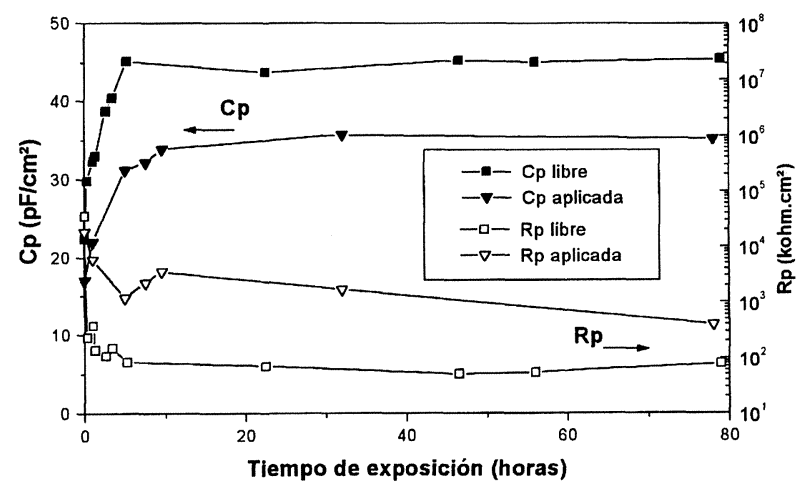

FIG. 4.- Evolución de la capacidad y la resistencia de la pintura, aplicada y libre.

FIG. 4.- Evolution with time of the capacitance $\left(C_{p}\right)$ and resistance $\left(R_{p}\right)$ for applied film (filled symbols) and free film (open symbols). 


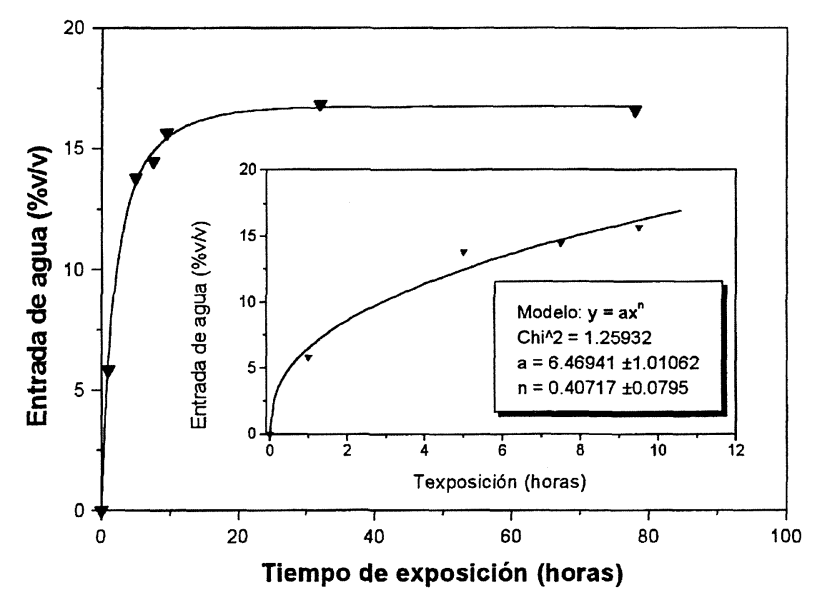

FIG. 5.- Entrada de agua en la película aplicada con el tiempo de exposición. En el recuadro aparece el ajuste exponencial del período inicial, previo a la saturación.

FIG. 5.- Water uptake for the applied film. The inserted plot presents the exponential fitting corresponding to the initial period, prior to saturation.

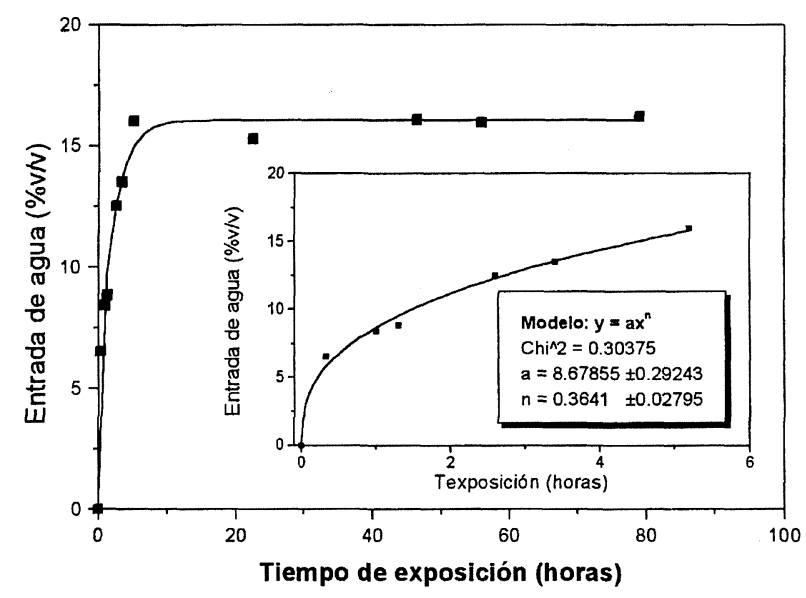

FIG. 6.- Entrada de agua en la película libre con el tiempo de exposición. En el recuadro aparece el ajuste exponencial del período inicial, previo a la saturación.

FIG. 6.- Water uptake for the free film. The inserted plot presents the exponential fitting corresponding to the initial period, prior to saturation.

etc.) que interaccionarán con el agua, también de forma distinta.

\subsection{Cálculo de los coeficientes de difusión}

Establecido que no es posible el cálculo del coeficiente de difusión mediante las leyes de Fick, se puede, no obstante, a partir del tiempo de saturación $\left(t_{\mathrm{s}}\right)$, obtener un valor aproximado del mismo, denominado coeficiente de difusión aparente, $D_{\mathrm{ap}}$ (11, 12 y 15):

$$
D_{\text {ap }}=\frac{L^{2}}{\tau}
$$

siendo $\tau$ el valor de la constante de tiempo de saturación de la película, que se puede identificar con el valor de $t_{s}$ obtenido en las gráficas 5 y 6, y $L$, el espesor de la película libre. Para el caso de películas aplicadas, el espesor se corresponde con la mitad del de la película libre en inmersión total, puesto que sólo existe un frente de entrada de agua. Sin embargo, la película libre empleada en nuestros experimentos poseía un único frente de entrada, al estar en contacto únicamente una de sus caras con la disolución. Por lo tanto, en ambos casos, se sustituyó $L$ por $2 d$, siendo $d$ el espesor de la película.

Teniendo todo esto en cuenta, los valores del coeficiente de difusión aparente de ambos sistemas son: $30,3 \cdot 10^{-9} \mathrm{~cm}^{2} / \mathrm{s}$ para la película aplicada y $14,8 \cdot 10^{-9} \mathrm{~cm}^{2} / \mathrm{s}$ para la película libre. Al comparar estos valores con los de la bibliografía (11 y 12 y 16 y 17), resultan superiores, aunque del mismo orden de magnitud. Este hecho no es sorprendente, hay que tener en cuenta que la pintura ensayada en este trabajo es una resina acrílica de base agua y, por tanto, poco resistente a dicho medio, mientras que los valores aparecidos en la bibliografía son de pinturas de base disolvente orgánico, más resistentes al agua, a pesar de lo cual, los coeficientes oscilan entre $10^{-11}$ y $10^{-9} \mathrm{~cm}^{2} / \mathrm{s}$.

\section{CONCLUSIONES}

Los resultados discutidos con anterioridad permiten concluir que:

- Los valores de capacidad obtenidos mediante la técnica de espectroscopía de impedancia resultan adecuados para el seguimiento de las propiedades dielétricas de la pintura.

- La cantidad de agua absorbida por la pintura es similar en ambos casos; sin embargo, la velocidad de entrada es menor en el caso de la pintura aplicada, por lo que ésta necesita un período de tiempo mayor para alcanzar la saturación, probablemente debido a la formación de una "película de agua" en la interfase soporte inerte-pintura.

- El ajuste exponencial de los datos experimentales de entrada de agua, durante la etapa previa a la saturación, conduce a la obtención de exponentes distintos de 0,5 , lo que resulta indicativo de un mecanismo de difusión no fickiano.

- Los valores de los coeficientes de difusión aparentes son significativamente mayores que los 
encontrados en la bibliografía para otros sistemas de pintura; este hecho refleja unas peores propiedades barrera.

\section{REFERENCIAS}

(1) Hansen, C.M. Prog. Org. Coat. 26, 1995:113-120.

(2) Belluci, F., Nicodemo, L., Monetta, T., Kloppers, M.J. y LATANision, R.M. Corr. Sci. 33 (8), 1992: 235-247.

(3) VAn Westing, E.P.M., Geenen, F.M., Ferrari, G.M. y de WIt, J.H.W. 9th European Corrosion Cong. Utrech (Países Bajos), 1989:CO-89.

(4) Walter, G.W. Corr. Sci. 32 (10), 1991: 1041-1058.

(5) Walter, G.W. Corr. Sci. 32 (10), 1991: 1059-1084.

(6) Shaw, G.S., Rogers, C.E., Payer, J.H. y Cuellar, E.A. J. Coat. Tech. 63 (795), 1991: 35-42.

(7) Walter, G.W. Corr. Sci. 32 (10), 1991: 1085-1103.
(8) Popov, B.N., Alwohaibi, M.A. y White, R.E. $J$. Electrochem. Soc. 140 (4), 1993: 947-951.

(9) LindQvist, S.A. Corrosion, 41 (2), 1985: 69-75.

(10) Brasher, D.M. y Kinsbury, A.H. J. Appl. Chem., 4, 1954:62.

(11) Kolek, Z. Prog. Org. Coat., 30, 1997: 287-292.

(12) Belluci, F., Nicodemo, L., Monetta, T., Kloppers, M.J. y LATANision, R.M. Corr. Sci. 33 (8), 1992: 1203-1226.

(13) Wind, M.M. y Lenderink, H.J.W. Prog. Org. Coat. 28 , 1996: 239-250.

(14) Nedler, J.A. y Mead, R. Computer J. 7, 1985: 308.

(15) Diguet, L. Tesis Doctoral: "Protection contre la corrosion par des revetements floures etude par voie electroquimique" París, 1996, p. 165.

(16) Miskovik-Stankovic, V.B., Drazic, D.M. y Teodorovic, M. J. Corr. Sci. 37(2), 1995: 241-252.

(17) Amidurin, A. y Thierry, D. Brit. Corr. J. 30 (2), 1995: 128-134. 